\title{
THz in Biology and Medicine: Towards Quantifying and Understanding the Interaction of Millimeter- and Submillimeter-Waves with Cells and Cell Processes
}

\author{
Peter H. Siegel ${ }^{a}$ and Victor Pikov \\ ${ }^{a}$ California Institute of Technology, Pasadena, CA 91125 USA \\ ${ }^{\mathrm{b}}$ Huntington Medical Research Institutes, Pasadena, CA 91105 USA
}

\begin{abstract}
As the application and commercial use of millimeter- and submillimeter-wavelength radiation become more widespread, there is a growing need to understand and quantify both the coupling mechanisms and the impact of this long wavelength energy on biological function. Independent of the health impact of high doses of radio frequency (RF) energy on full organisms, which has been extensively investigated, there exists the potential for more subtle effects, which can best be quantified in studies which examine real-time changes in cellular functions as RF energy is applied. In this paper we present the first real time examination of RF induced changes in cellular activity at absorbed power levels well below the existing safe exposure limits. Fluorescence microscopy imaging of immortalized epithelial and neuronal cells in vitro indicate increased cellular membrane permeability and nanoporation after short term exposure to modest levels (10-50 $\mathrm{mW} / \mathrm{cm}^{2}$ ) of RF power at $60 \mathrm{GHz}$. Sensitive patch clamp measurements on pyramidal neurons in cortical slices of neonatal rats showed a dramatic increase in cellular membrane permeability resulting either in suppression or facilitation of neuronal activity during exposure to sub- $\mu \mathrm{W} / \mathrm{cm}^{2}$ of RF power at $60 \mathrm{GHz}$. Non-invasive modulation of neuronal activity could prove useful in a variety of health applications from suppression of peripheral neuropathic pain to treatment of central neurological disorders.
\end{abstract}

Keywords: Millimeter Waves, Cells, Neuronal Activity, Non-Invasive

\section{INTRODUCTION}

The increasingly widespread use of millimeter-wave generators for wireless telecommunications [1], security screening [2], and even non-lethal crowd control weapons [3], should have prompted renewed scientific interest in the effects of this wavelength range on biologic materials and organisms. Yet with no dedicated funding from U.S. Civilian Federal Agencies, very few research groups are willing to undertake these studies. Several investigations [4-16], mainly outside the U.S., have noted significant impact on neuronal activity from modest level millimeter wave exposures (40-130 GHz, $1-100 \mathrm{~mW} / \mathrm{cm}^{2}$, seconds to minutes) that are not much higher than the Federal Communications Commission-established maximum permissible exposure (MPE) limits of $1 \mathrm{~mW} / \mathrm{cm}^{2}$ for 6 minutes in the 30-300 GHz frequency regime [17]. Synchronization of the firing rate of neurons in the hypothalamus of both rabbit and rat was observed at and below 10 $\mathrm{mW} / \mathrm{cm}^{2}[4,5] .53 \mathrm{GHz}$ exposure of the sciatic nerve in rats at only $4 \mathrm{~mW} / \mathrm{cm}^{2}$ increased the action potential amplitude [6]. Even at $2-3 \mathrm{~mW} / \mathrm{cm}^{2}$, at certain frequencies between 40 and $52 \mathrm{GHz}$ an isolated frog sciatic nerve showed measureable changes in the amplitude and latency of its compound action potential [7]. Higher levels of millimeter-wave power $\left(10-100 \mathrm{~mW} / \mathrm{cm}^{2}\right)$, which tend to raise the temperature of the exposed sample, have been shown to produce changes in neuronal activity that sometimes do, and sometimes don't correlate with broadband radiant heating [8-11]. For example, changes in action potential firing rates in skate skin exposed to $130 \mathrm{~mW} / \mathrm{cm}^{2}$ at $54 \mathrm{GHz}$ were anticorrelated with those produced by radiant heating [8]. In another study, however, exposure of snail pacemaker neurons to $75 \mathrm{GHz}$ radiation at levels sufficient to raise the temperature several degrees in a few seconds showed changes in firing rate that matched those produced by radiant heating [9]. Similar results correlating millimeter wave exposure (62 and $75 \mathrm{GHz}$ ) and direct temperature rise were also reported for the changes induced in ionic currents in these neurons [10]. Most recently, the electrical response of an exposed frog sural nerve ( $>45 \mathrm{~mW} / \mathrm{cm}^{2}$ at $\left.42 \mathrm{GHz}\right)$ showed threshold effects and transient behavior that were not well reproduced by broadband radiant heating [11]. Additional studies have focused on millimeter-wave induced changes in cell membrane permeability. Small increases in current transport across lipid bilayers were seen with both pulsed and continuous wave $(\mathrm{CW})$ millimeter wave power between 54 and $76 \mathrm{GHz}$

Optical Interactions with Tissues and Cells XXI, edited by E. Duco Jansen, Robert J. Thomas,

Proc. of SPIE Vol. $7562,75620 \mathrm{H} \cdot$ @ 2010 SPIE · CCC code: 1605-7422/10/\$18 $\cdot$ doi: $10.1117 / 12.845380$

Proc. of SPIE Vol. 7562 75620H-1 
[12]. Similarly, the permeability of phospholipid based liposomes increased after exposure at $130 \mathrm{GHz}$ with 10$17 \mathrm{~mW} / \mathrm{cm}^{2}$ [13]. Annexin V, an extracellularly-applied marker, was used to visualize the outward and inward migration of the membrane-forming lipid, phosphatidylserine, during exposure of keratinocytes to $42 \mathrm{GHz}$ at $35 \mathrm{~mW} / \mathrm{cm}^{2}$ [14]. Radiant heating of the cells failed to reproduce the effect. Finally, in a set of experiments on mouse skin receptors, the tail flick response was observed to decrease with millimeter wave exposure $[15,16]$ in contrast to simple heating.

The capability of millimeter waves to induce both excitatory and inhibitory responses in neurons as well as the potential to induce changes in membrane permeability at power levels that are insufficient to cause dramatic changes in the local thermal environment warrant a more detailed look at the energy coupling occurring at the cellular level. Recent investigations on the prevalence and role of macromolecule-bound water, particularly inside and adjacent to the cellular membrane, point to strong specific absorption in the millimeter wave band [18-21]. The authors' intent in this paper is to describe several experiments, performed in vitro and ex vivo, which address the impact of millimeter waves on individual cells at the microscopic level. In the course of these investigations, we found evidence for transient membrane depolarization in epithelial and neuronal cell lines and altered neuronal firing in rodent cortical slices at RF power levels well below the MPE.

\section{EXPERIMENTS}

\subsection{Instrumentation}

Our microscopic setup for real time measurement of cellular functions during millimeter wave exposure is shown in Fig. 1. An inverted tissue culture microscope (Nikon Diaphot 300) serves as a flexible observation platform allowing RF energy to be injected from above the sample. Epifluorescent illumination with specific laser lines or a broad band halogen lamp, and microscopic viewing were accomplished from below the sample. Focused white light, used for visual inspection and alignment, was introduced from above, either through the in-line focus illuminator or from the side to bypass the RF injection waveguide. CCD photography or video recording of the visual field was accomplished from a side port of the Diaphot. Spectral data was collected using a front port usually intended for a $35 \mathrm{~mm}$ camera mount, by introducing an aperture and short NA objective that focuses into a multimode fiber. Standard dichroic filter cubes were introduced below the sample stage and above the objective turret, and additional single filters were sometimes installed in the rear epifluorescent tube, at the oculars or in front of the spectral port objective. Single mode, linearly polarized RF power was supplied by an open ended waveguide placed above the sample and coupled to a calibrated attenuator, a directional coupler or waveguide switch (for continuous power monitoring via an HP436A microwave power meter and appropriate sensor head), an isolator, and a variety of commercial and custom RF generators. RF power was modulated through the generator electronics, a waveguide ferrite modulator or via a simple optical chopper wheel that can be inserted into a small gap in the RF waveguide path. We have used a carcinotron, backward wave oscillator, Gunn and Impatt diode oscillators and upconverted microwave oscillators coupled with millimeter wave power amplifiers as sources in the 40-140 GHz frequency range. The most challenging procedure was the characterization of the power density and specific absorption rate (SAR) applied to the sample. Since most culture media are water based, and the RF absorption coefficient, $\alpha$, in pure water at $60 \mathrm{GHz}$ for example is approximately $50 \mathrm{~cm}^{-1}$ with a refractive index close to 4 , a variation of a few microns in the fluid depth between the sample and the air filled waveguide causes substantial changes in coupling of the incident power. Ideally one would like to place a matched and immersed calorimeter at the sample plane, but this was not possible as no such commercial calorimeters exist for the frequency range employed. In order to mitigate this problem we introduced a culture chamber (Fig 2), the Nunc Opticell ${ }^{\mathrm{TM}}$ Culture System (BioCrystal Ltd.), which consists of two gas permeable polystyrene membranes separated by a $2 \mathrm{~mm}$ gap and can be repeatedly filled and emptied with $10 \mathrm{ml}$ of fluid. Due to the water-tight seal of the Opticell chamber, it was possible to flip the chamber prior to placement under the microscope, so that the cultured cells that were adhered on the bottom membrane were now facing the RF beam above, with only a thin $(75$ micron) polystyrene sheet in between, i.e. no intervening water. The low loss and low permittivity of the polystyrene in the millimeter wave region allowed most of the available power from the RF source to reach the sample. With knowledge of the beam properties of the waveguide launcher and the distance to the Opticell surface, it was possible to calculate with reasonable certainty the incident RF energy at the plane of the cells. 

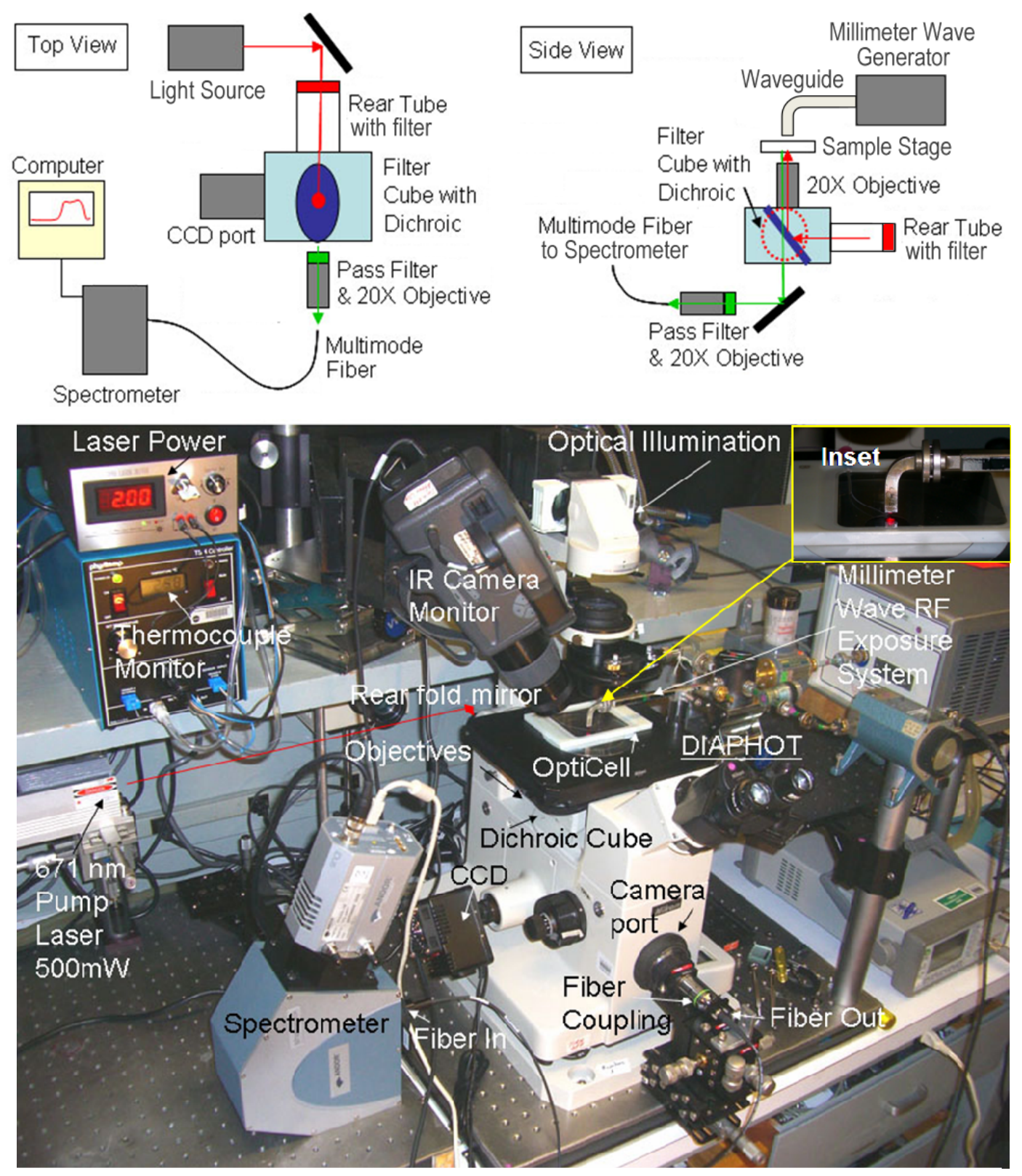

Fig. 1. Schematic (above) and photograph (below) of experimental set up used for RF exposure and real time monitoring of in vitro response of cells. Includes epifluorescence, Raman and optical spectroscopy and IR camera and thermocouple temperature sounding as well as a heated stage. Photomultiplier tubes (red and green filtered) are placed in the oculars when needed. RF exposure is from the top via open ended millimeter waveguide or Gaussian mode horn. Power is incident from various oscillators and monitored with a frequency meter, calibrated power meter and calibrated variable attenuator. An optical chopper can be added in the RF path. Optical to fiber coupling for the spectrometer occurs at the $35 \mathrm{~mm}$ camera port. Laser illumination through the rear epifluorescent port replaces the halogen lamp when needed. 


\subsection{Response to High Power Exposures}

For our initial series of experiments we cultured an immortalized epithelial cell line designated H1299 (a gift from Dr. Alex Sigal, Caltech division of biology), in both standard polystyrene Petri dishes and the Opticell culture chamber. The H1299 cells were fairly large and strongly adhered to the walls of both culture chambers (Fig. 3). The cells were incubated in $5 \% \mathrm{CO}_{2}$ at $37^{\circ} \mathrm{C}$ in RPMI-1640 medium (Roswell Park Memorial Institute) supplemented with $10 \%$ fetal bovine serum and $2 \mathrm{mM}$ L-glutamine. For most of the RF exposures, the RPMI was replaced with PBS (phosphate buffered saline) to better enable subsequent epifluorescent and spectroscopic measurements. Using the test set up of Fig. 1 , the cell chamber was allowed to equilibrate with ambient room temperature $\left(23^{\circ} \mathrm{C}\right)$, inverted, and subjected to $\mathrm{RF}$ illumination, while the surrounding bath temperature was monitored through an infrared camera (Inframetrics Thermacam PM290, FLIR Systems). Under exposures of approximately $300 \mathrm{~mW} / \mathrm{cm}^{2}$ at $60 \mathrm{GHz}$ for two minutes we noticed substantial heating of the culture media $\left(>7^{\circ} \mathrm{C}\right)$ and cell movement away from the exposed area over a period of 10-20 minutes. Using Annexin V conjugated to $\mathrm{Cy} 3$ as a fluorescent marker of membrane pores, we noted no permanent pores at 18 hours after the exposure. At an increased power density level of $700 \mathrm{~mW} / \mathrm{cm}^{2}$ and the same two minute exposure time, the whole exposed area under the waveguide was positive for Annexin V staining at 18 hours, indicating the formation of permanent cellular membrane pores indicative of cell apoptosis (Fig. 4). However, even at this high exposure level, the bath temperature was below $40^{\circ} \mathrm{C}$, as recorded with the IR camera.

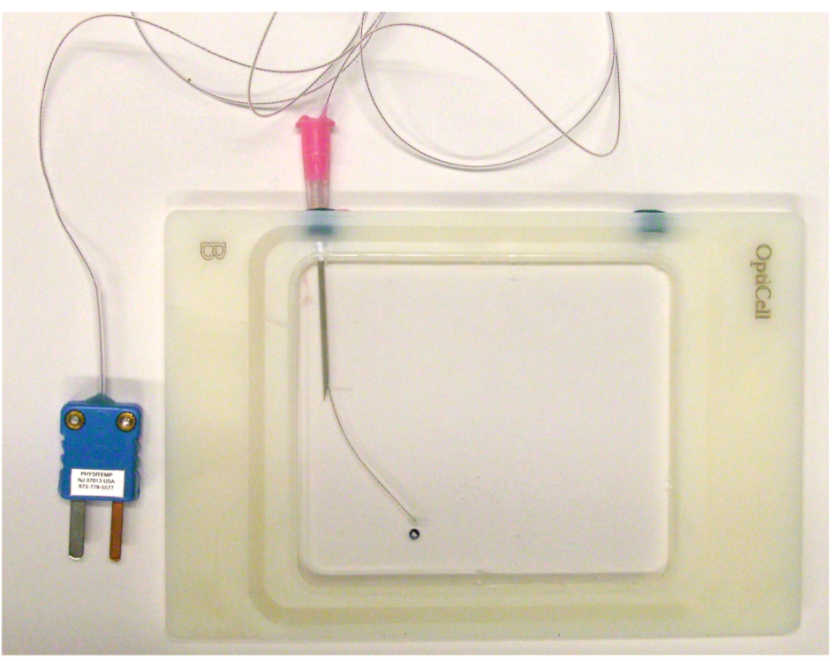

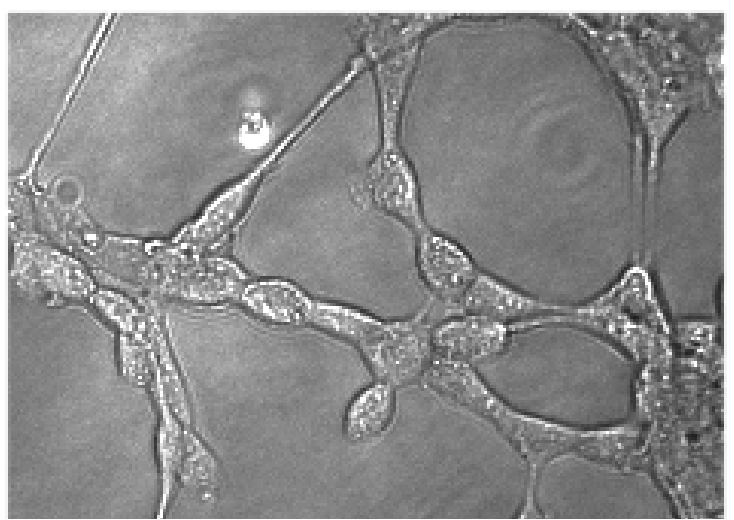

Fig. 3. H1299 epithelial cells cultured in resealable polystyrene dishes. Incubated in RPMI at $37 \mathrm{C}$ in $5 \% \mathrm{CO}_{2}$ and imaged at $200 \mathrm{X}$

Fig. 2. Photograph of the Opticell culture chamber used for RF exposures and Raman temperature measurements on the H1299 epithelial cells. The field of view is shown by the small black circle with the thermocouple immediately outside. The chamber is water tight, bounded by thin gas permeable polystyrene sheets $2 \mathrm{~mm}$ apart, and holds $10 \mathrm{ml}$ of fluid.

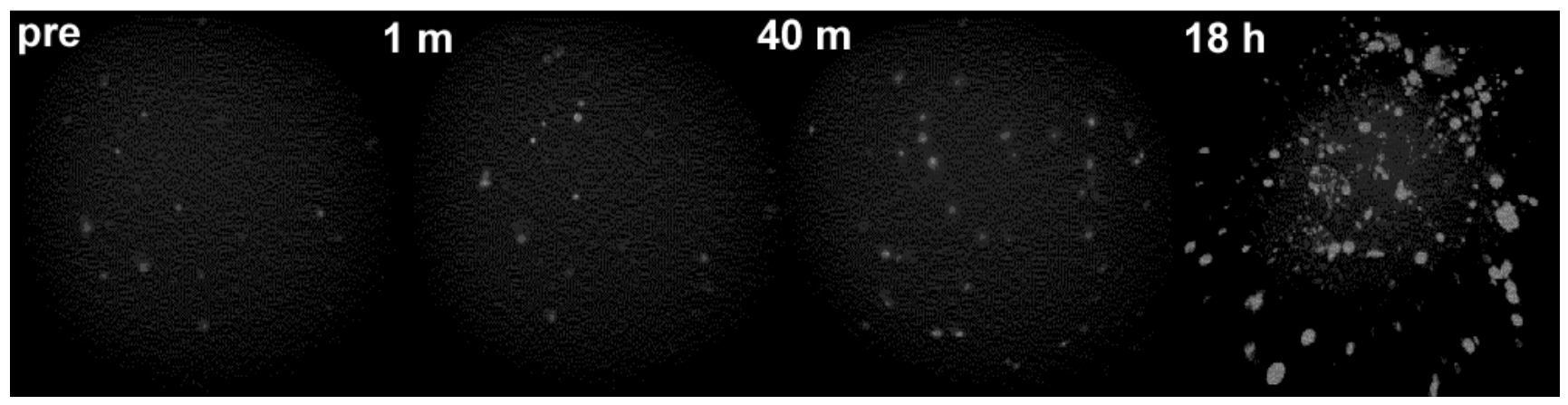

Fig 4. Cell apoptosis after RF exposure at $60 \mathrm{GHz}$ for 2 minutes at $700 \mathrm{~mW} / \mathrm{cm}^{2}$ 


\subsection{Non-Contact Temperature Monitoring of Cells}

Next, we examined the question of whether the temperature of the cells exceeded that of the bath during the RF exposures and hence contributed to subsequent cellular apoptosis. We considered using an IR microscope, but were concerned with the accuracy of readings when the sample is located at some depth under a layer of culture media. A second alternative would be to use a microthermocouple, however, there was a concern that the metallic probe would interfere with the millimeter-wave induced RF fields in and around the sample and affect the absorbed power. Therefore, we chose to develop a novel non-contact optical method based on a near-IR Raman spectroscopy technique. The combination of several vibrational modes in hydrogen $(\mathrm{O}-\mathrm{H})$ bonds of water gives rise to a broad spectral resonance in the 3200-3700 $\mathrm{cm}^{-1}$ range. The strengths and frequencies of the resonance peaks are strongly dependent on temperature in the biologically relevant range of 0 to $50^{\circ} \mathrm{C}$ [22]. Kakuta et.al. [23] utilized these $I R$ spectral absorption signatures to correlate the temperature of a microscopic water sample with changes in the transmission amplitude and peak frequency of the O-H vibrational modes. Smith et.al. [24] suggested a two component Gaussian fitting technique to accurately reproduce this spectral envelope. We chose to apply a related approach of Raman spectroscopy which, unlike the IR absorption, can be used at visible and near-IR wavelengths, compatible with epifluorescent microscopy. Cells were excited by a $671 \mathrm{~nm}$ diode pump laser, and the Raman spectrum of the hydrogen vibrational modes was seen in the range from 800 to $900 \mathrm{~nm}$, just below the upper transmission limit of standard optical objectives and well above the nonspecific biological autofluorescent band $(<600 \mathrm{~nm})$. Using the setup shown in Fig. 1, the backscattered $671 \mathrm{~nm}$ laser light and most of the backscattered Rayleigh light are rejected by the dichroic cube filter $(>700 \mathrm{~nm}$ pass) while the Raman signal in the $800-900 \mathrm{~nm}$ spectral range was allowed to pass into the $20 \mathrm{X}$ objective at the front port of the microscope. A second inline, long wavelength pass filter ( $>785 \mathrm{~nm}$ pass) blocked any residual Rayleigh or laser light before the signal was focused into a multimode fiber and sent to a spectrometer (Shamrock SR 163, Andor). Raman spectra of DI water, cells in RPMI media and cells in PBS were measured as the temperature was changed from 25$40^{\circ} \mathrm{C}$. (Figs. 5 and 6). A two peak fitting algorithm was used to separate the Raman signal into two curves, and the areas under the two peaks were compared. The ratio of the areas was then used to correlate with the measured temperature using an in-situ thermocouple (Fig. 7). Raman spectroscopy also allowed determination of focal position, or depth, within the culture chamber. This was possible due to the appearance of two distinct and relatively narrow Raman peaks of polystyrene at 2950 and $3150 \mathrm{~cm}^{-1}$ (Fig, 8), appearing on the left side of the water signature. As the focus of the excitation laser (objective lens) moved from the first polystyrene membrane, through the fluid, and on to the second polystyrene membrane, these peaks allowed us to calibrate the vertical scale. Finally, it was possible to record the temperature rise of individual cells in PBS when exposed to modest levels (up to $100 \mathrm{~mW} / \mathrm{cm}^{2}$ ) of millimeter wave power for 2 minutes. The Raman-based measurement of cellular heating indicated that cellular temperature was higher than that of the surrounding media, recorded both by a commercial IR camera and an embedded thermocouple [25].

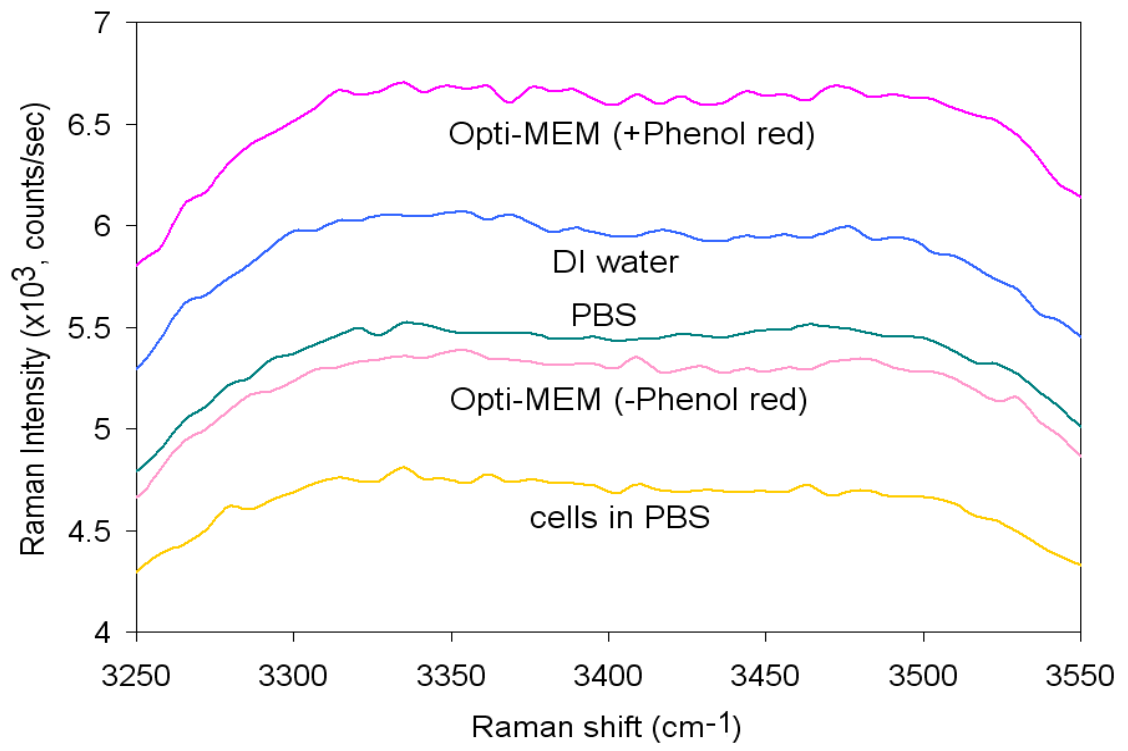

Fig 5. Raman spectra of various water-based media inside the OptiCell dish at $25^{\circ} \mathrm{C}$. Although changes in total signal are apparent due to the amount of Raman backscatter, the spectral line shape is very consistent. 


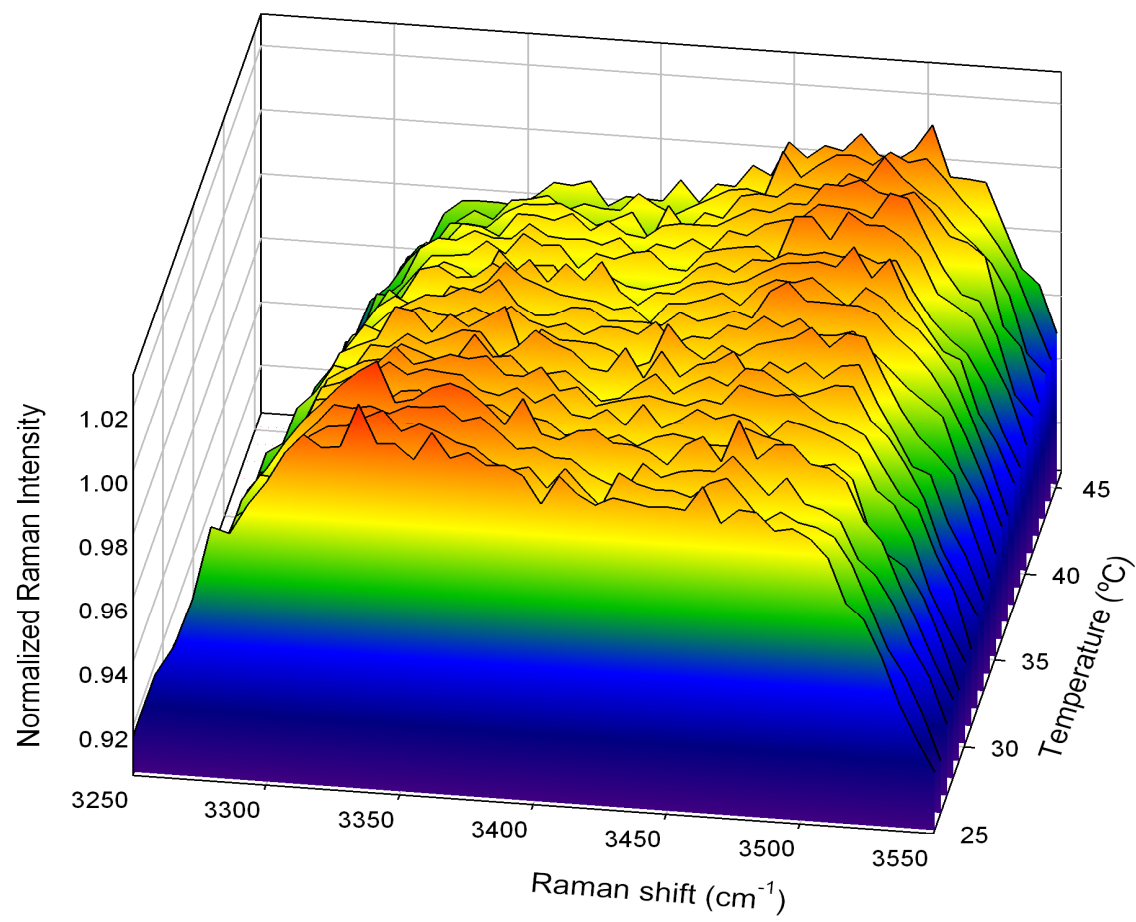

Fig. 6. Temperature dependence of Raman spectrum in H1299 immortalized epithelial cell line between 25.2 and $44.2^{\circ} \mathrm{C}$ at $1{ }^{\circ} \mathrm{C}$ intervals. The cells were cultured in RPMI medium in OptiCell dish at $37^{\circ} \mathrm{C}$ and $5 \% \mathrm{CO} 2$. For spectroscopy measurement, the RPMI was replaced with PBS.

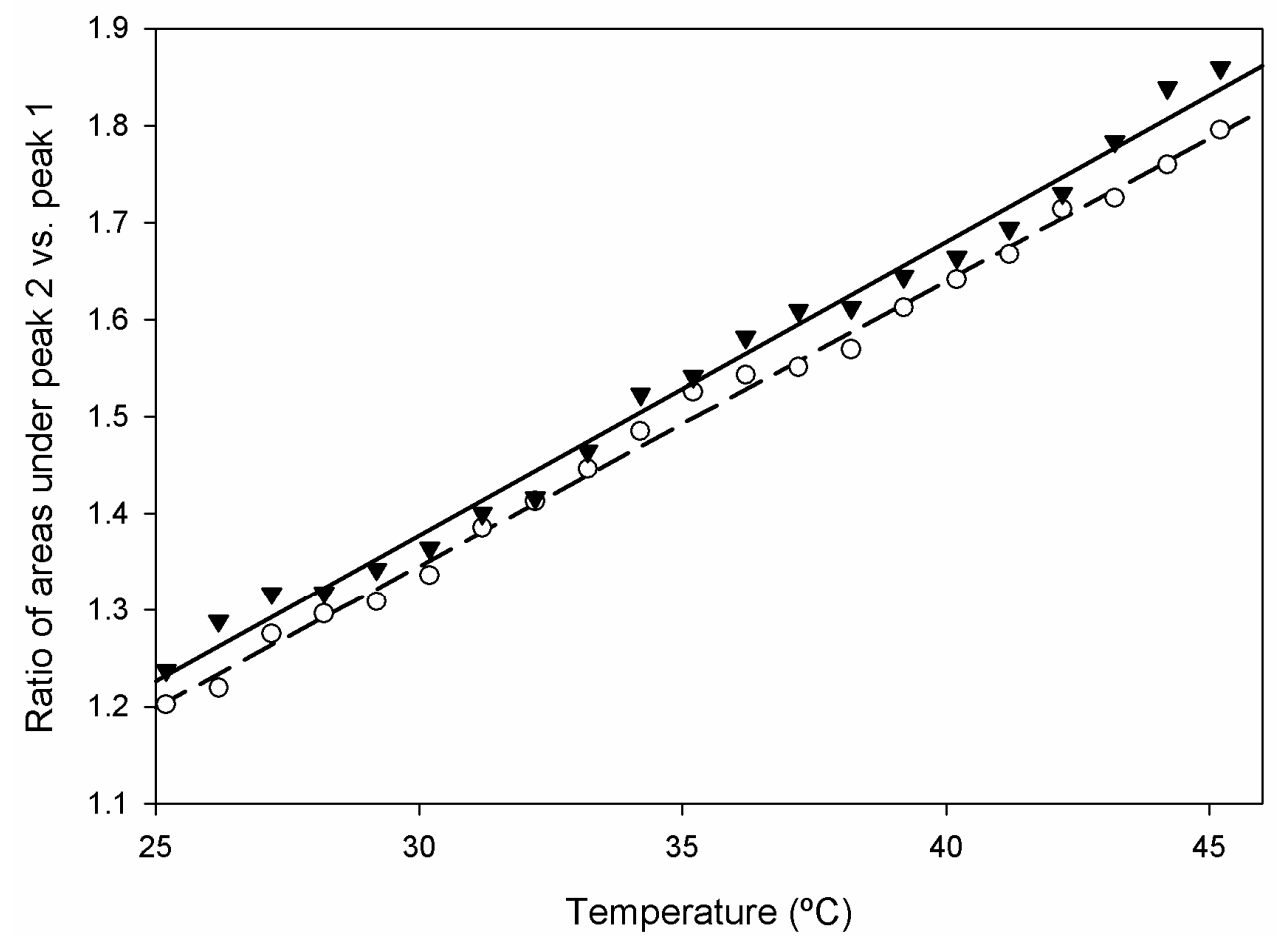

Fig. 7. Fit of the changes in measured spectral shape (peak area) of the $\mathrm{OH}$ bands of DI water (open circles) and $\mathrm{H} 1299$ cells in PBS (solid triangles) at temperatures between 25.2 and $44.2^{\circ} \mathrm{C}$ in an OptiCell culture system. Linear regression for the DI water data $\left(\mathrm{R}^{2}=0.996\right)$ is shown as a dotted line and for the H1299 cells $\left(\mathrm{R}^{2}=0.990\right)$ as a solid line. 


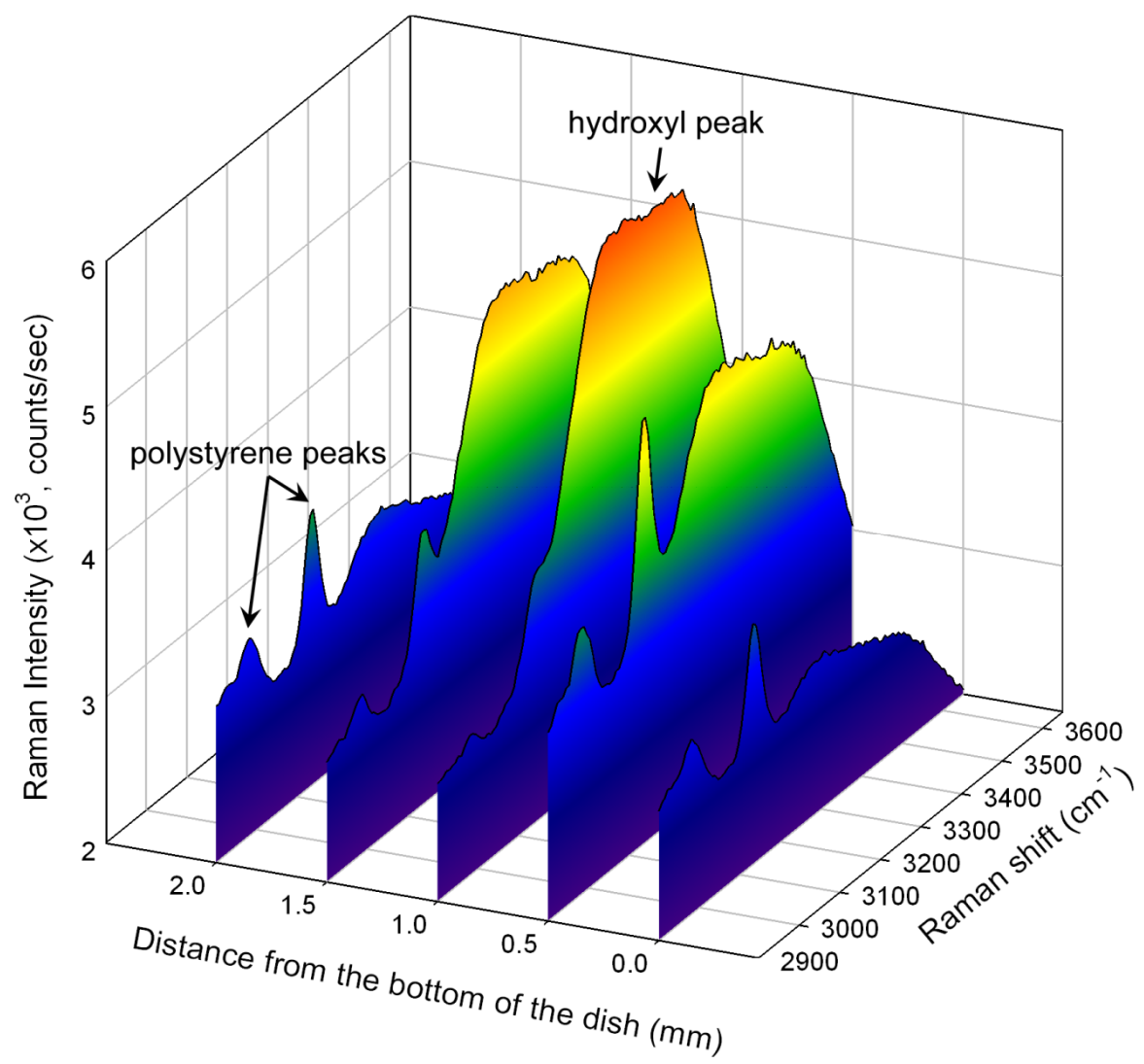

Fig. 8. Raman spectra showing progression of focal plane from the bottom, through $2 \mathrm{~mm}$ of water, and to the top of OptiCell culture system.

\subsection{Affecting Cellular Membrane Permeability In Vitro}

The temperature measurements in the H1299 cells suggested a slight concentration of absorbed energy in the cell relative to the surrounding media. Extrapolating from the large body of work on enhanced slow relaxation vibrational mode density for water in protein solvates $[18,19$ e.g.] and recent observations of increased absorption in macromoleculebound water at $\mathrm{THz}$ frequencies [20,21], we hypothesized that millimeter wave absorption may occur at the cellular membrane. We employed a non-invasive fluorescent microscopy approach to look for real time changes in membrane permeability on exposure to millimeter waves on a multi-second timescale. To produce the fluorescent marker of membrane permeability, the plasmid containing farnesylated green fluorescent protein (pEGFP-F), was transfected into the cell membrane of H1299 cells, and its expression was stabilized through a standard antibody resistance selection process over a period of approximately 6 weeks. The farnesylation signal on the fGFP protein was used to target it to the inner leaflet of the cellular membrane (Fig. 9) where it fluoresces at $520 \mathrm{~nm}$ (green) upon exposure to $490 \mathrm{~nm}$ (blue) light. Using a method developed recently by DiFranco et.al. [26], oxonol dye (Invitrogen DiBAC 4 (5)) was added to the media where it concentrated on the outer layer of the membrane of the cells. The excess oxonol was removed by changing out the medium, and the bound oxonol then served as a quenching agent for the fGFP signature through a FRET (Förster resonance energy transfer) process. During membrane depolarization, oxonol migrates into the inner leaflet, approaching the bound fGFP, and fGFP emissions are quenched (absorbed) by the oxonol and re-emitted as red (620nm) fluorescence. Thus, the shift from green to red fluorescence indicates the increase in cellular permeability leading to depolarization. 
Prior to the exposure, RPMI medium in the Petri dish was replaced with PBS containing oxonol at a concentration of $5 \mu \mathrm{M}$ for one minute. The medium was then replaced with , a thin layer $(<1 \mathrm{~mm})$ of pure PBS solution above the adhered cells. The open ended waveguide was positioned directly above the fluid and RF power at $50 \mathrm{GHz}$ was introduced at an approximate density of $1-3 \mathrm{~mW} / \mathrm{cm}^{2}$ at the cells for two minutes. Excitation light from the halogen source was passed through a narrowband filter $(477-495 \mathrm{~nm}$, Omega XF1042) and focused onto a single cell in the field of view (approximately 20 microns in diameter). The backscattered fluorescent signal was sent through a 500nm high-pass dichroic mirror (Omega XF2031), passed through a 10X microscope objective and collected at the front port of the microscope. The light was then focused via a 20X objective into a multimode fiber and sent to the spectrometer, in this case an uncooled HR2000 (Ocean Optics). The poor sensitivity of this spectrometer required a rather long integration time of 40 seconds, while the data was collected continuously over a period of several minutes before, during and after $\mathrm{RF}$ exposure(Fig. 10). Before the RF exposure, the spectral signature peaks in the green wavelength region consistent with the direct GFP fluorescence. During the two-minute RF exposure a strong red signal appears, attributed to the FRET oxonol signature and indicating the membrane depolarization. After six minutes, the cell appears to return to the baseline fluorescent state, i.e. signaling the re-polarization of the membrane and the return of the oxonol to the outer leaflet.
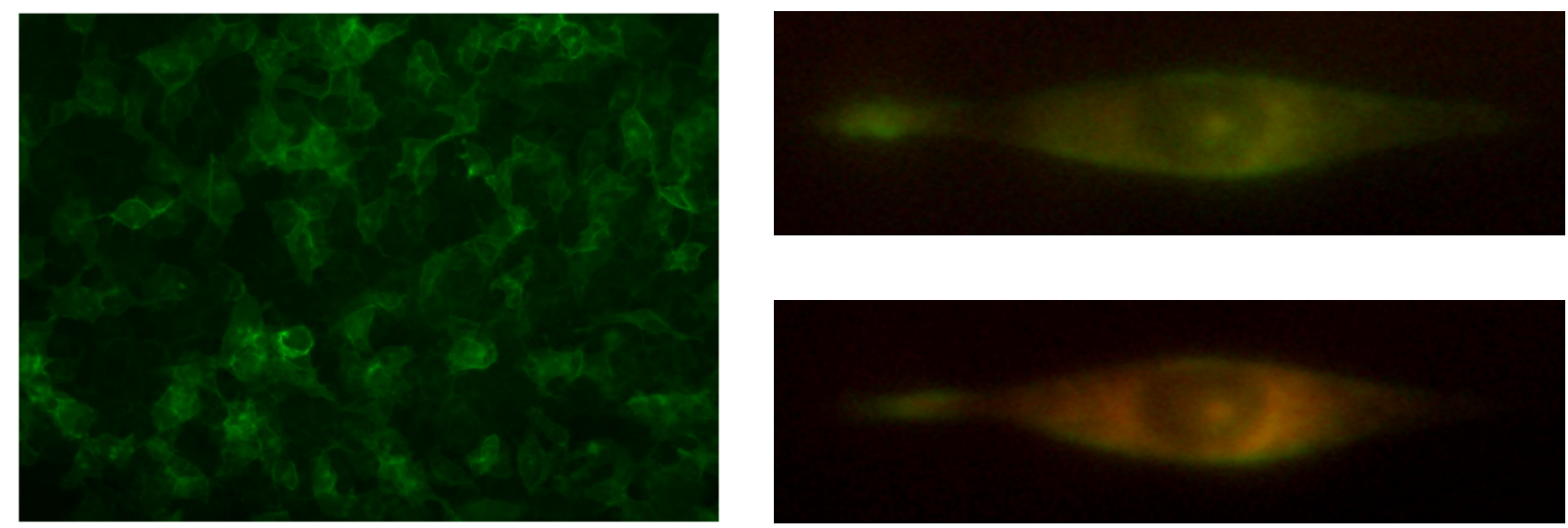

Fig 9. Left. Photograph of H1299 cell field expressing lipid bound fGFP at cell membrane (transfected with pEGFP-F vector). Right. Photograph of single fGFP expressing cell in oxonol and PBS before (top) and during (bottom) millimeter wave exposure. Red color in the bottom picture indicates FRET-induced oxonol fluorescence.

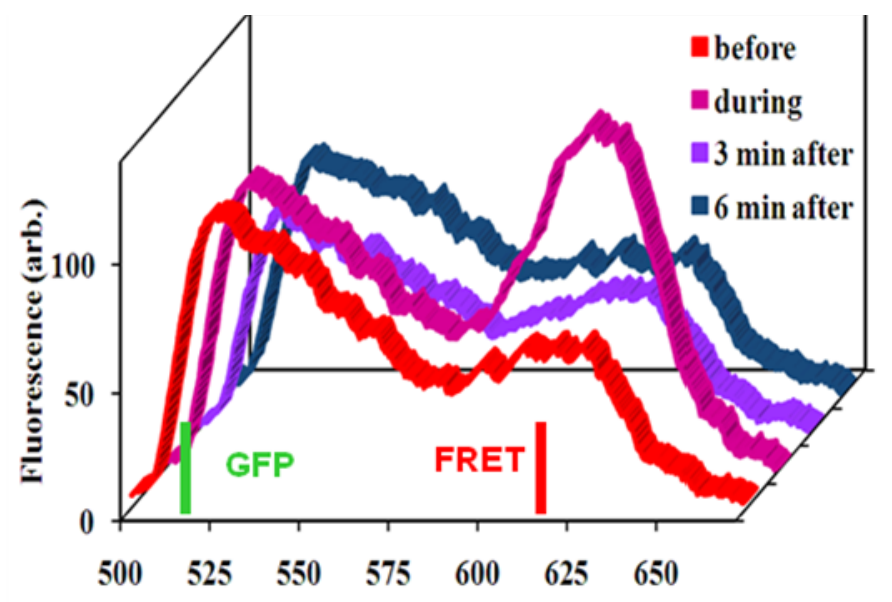

Frequency $(\mathrm{Hz})$

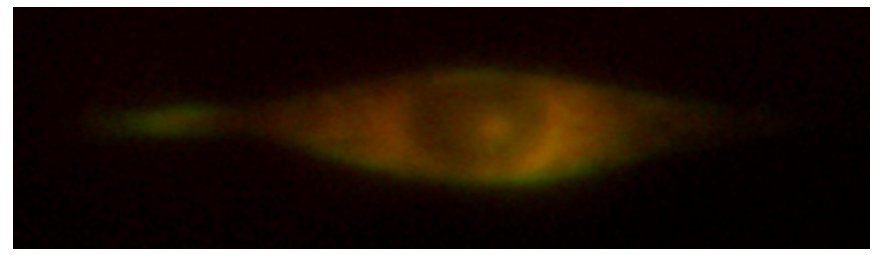




\subsection{Affecting Neuronal Permeability In Vitro}

In addition to H1299 cells, a neuronal cell line B104 (a gift from Peter W. Vanderklish, Scripps Institute) was transfected with fGFP using a plasmid and the expression was stabilized over ten weeks (Fig. 11).

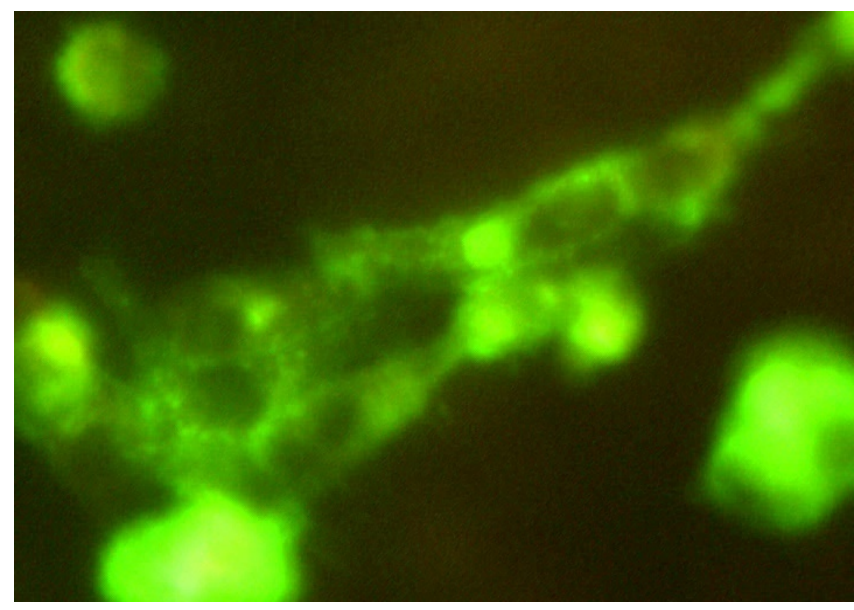

Fig.11. Neuronal cell line transfected with pEGFP-F and expressing fGFP in the membrane.
In order to reduce the acquisition time for the FRET signal, we placed two sensitive photomultiplier tubes (Hamamatsu H5784-20 PMT) in the binocular focus tubes of the microscope for red/green differential amplitude measurement. In front of one of the two PMTs, we placed a red band pass filter (3RD610-650, Omega), and in front of the other a green filter (3RD510-550, Omega). PMT signals were digitized and continuously recorded through a $\mathrm{LabView}^{\mathrm{TM}}$ program. The CCD camera (TCC-3.3ICE-N, Tucsen Imaging Technology) was used for coincident monitoring of cellular location. PMT measurements on the B104 cells did not fully replicate our earlier findings in the H1299 cells during exposures to similar RF power densities $\left(10 \mathrm{~mW} / \mathrm{cm}^{2}\right.$ at $60 \mathrm{GHz}$ ). Although there was an observed shift from green to red fluorescent signature, indicating the membrane depolarization, no return to baseline, indicating repolarization, was observed. Subsequent discussions with Professors Vergara and DiFranco (UCLA) pointed toward possible enhanced solubility of oxonol in PBS upon millimeter-wave exposure resulting in a permanent increase in the red background signal. A typical PMT measurement, shown in Fig. 12, indicates a sustained FRET process (increase in the red channel). The observed decrease in the red signal seen in the figure was attributed to photobleaching.

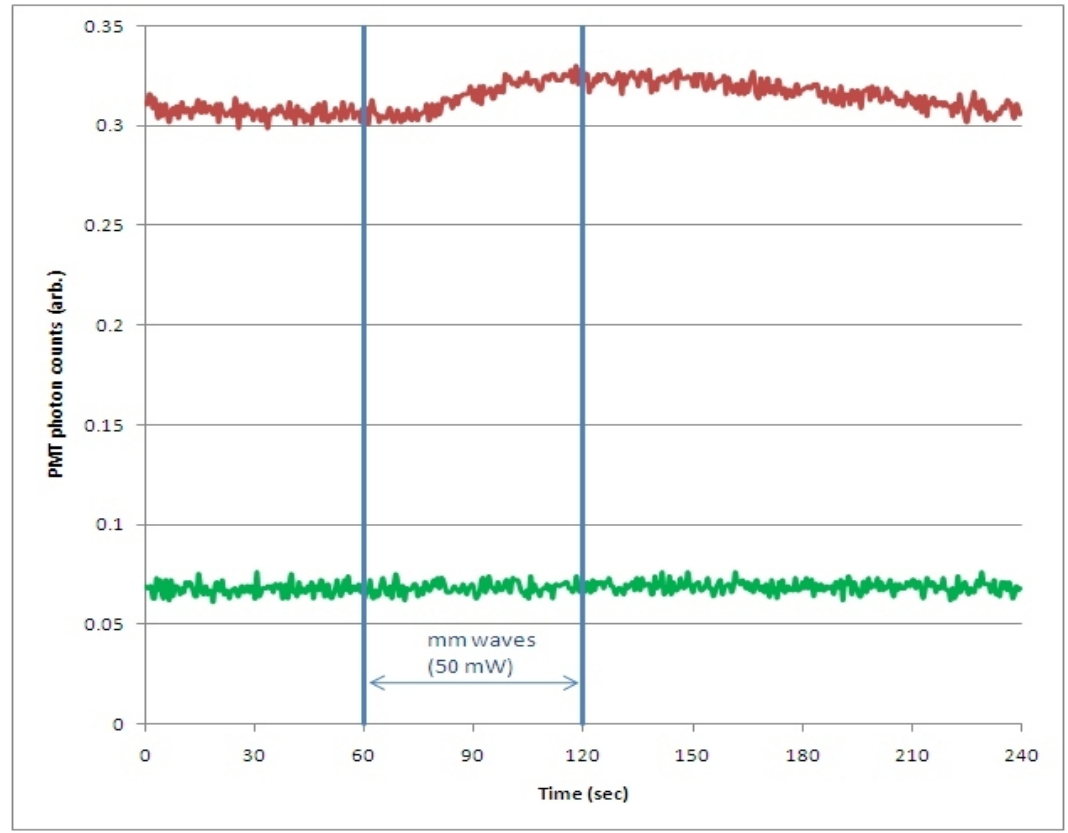

Fig.12. PMT signals before (left), during (between the vertical lines) and after (right) exposure of the neurons to RF $\left(10 \mathrm{~mW} / \mathrm{cm}^{2}, 60 \mathrm{GHz}\right)$ 


\subsection{Affecting Neuronal Permeability and Activity Ex Vivo}

Difficulties associated with indirect optical measurements of the membrane potential led us to an experiment involving direct electrophysiological measurement of neuronal activity (Fig. 13). An upright Nikon FN1 microscope fitted with a continuously perfused turbulence-free laminar flow tissue chamber (Cell MicroControls BT-1-18) and a precision micromanipulator holding a pulled glass electrode (World Precision Instruments) was interfaced to a MultiClamp 700B current amplifier and Digidata 1440 digitizer controlled via a PC running pCLAMP software (Molecular Devices). RF power of up to $185 \mathrm{~mW}$ at $60 \mathrm{GHz}$, derived from a solid state injection locked Impatt oscillator, was introduced through the same waveguide as in the earlier experiments. Power was continuously monitored through a directional coupler and HP436A power meter. The power level was set by a mechanical vane attenuator, which is the only knob that is turned during the measurements. No electronic switches were used. Rat pups, 13 and 16 days old, were anesthetized and decapitated and their brains perfused in ice cold artificial cerebrospinal fluid (ACSF). 300 micron thick vibrotome slices containing the cerebral cortex were placed in the tissue chamber and incubated at room temperature in the ACSF (approximately $3 \mathrm{ml}$ ) with bubbled oxygen $(95 \%)$ and $\mathrm{CO}_{2}(5 \%)$ at a $\mathrm{pH}$ of 7.4. The probe pipette was filled mostly with K-glutonate, $\mathrm{KCl}$ and $\mathrm{NaCl}$ (140:5:4) plus a buffer agent (HEPES) to obtain a resistance between 4 and $7 \mathrm{M} \Omega$. The ionically balanced probe was inserted through the membrane and into the head of a single pyramidal neuron and positive current (40-200 pA) was applied for five seconds out of every twenty seconds, repeating cyclically for several minutes during each measurement sequence. The intracellular-to-extracellular voltage was recorded continuously and the appearance, firing rate, peak voltage, rise and fall times and input resistance $(\mathrm{dV} / \mathrm{dI})$ of the action potential spikes upon the positive current cycles provided the baseline and RF induced changes that are the basis for the experiments. RF power $(60 \mathrm{GHz}$, with $7.5,15,30,60,120$ and $185 \mathrm{~mW}$ present at the waveguide output port) was introduced in random sequence via the attenuation setting, held fixed for one minute ( 3 current cycles), then turned off. Bath temperature was constantly monitored by an in-situ thermocouple. The temperature rise with power level varied from $<0.1^{\circ} \mathrm{C}$ to a maximum of $3^{\circ} \mathrm{C}$ at the highest setting. Fig. 14 shows sample recordings taken at four different RF power levels from approximately 0.1 to $1 \mu \mathrm{W} / \mathrm{cm}^{2}$ as calculated at the plane of the cortical slice after absorption through $2.2 \mathrm{~mm}$ of ACSF. Changes in the firing rate were observable at power levels of $\sim 0.3 \mu \mathrm{W} / \mathrm{cm}^{2}$ and above. Rise and decay slopes of individual action potentials (Fig. 15) and membrane resistance (not shown) were also strongly correlated with RF power level indicating opening of the membrane ion channels. These experiments are described in greater detail in [27].
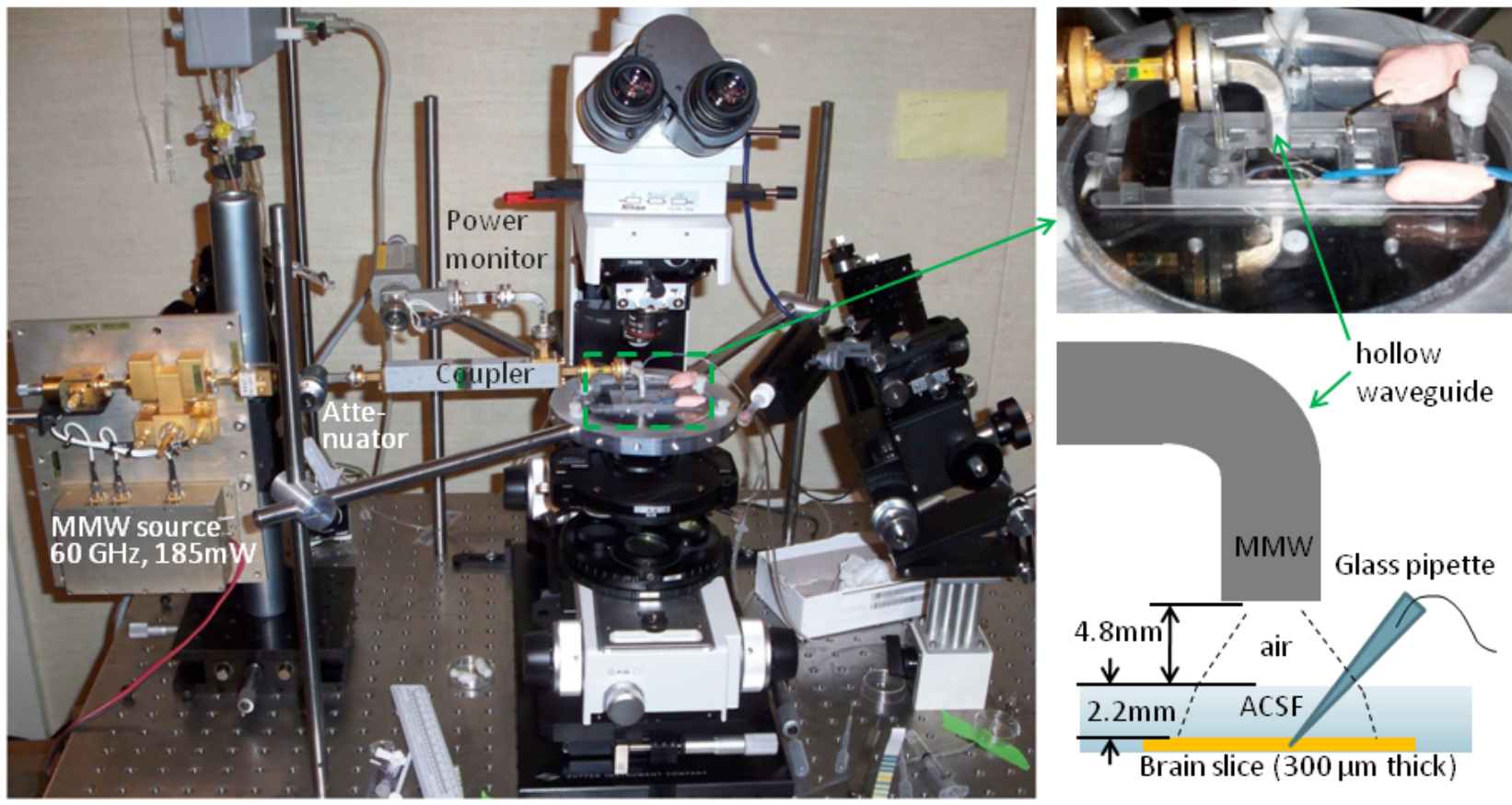

Fig. 13. Left. Photograph of patch clamp measurement set up with vertically coupled RF power source. Above right. Close up showing waveguide and tissue chamber. Below right. Schematic showing probe geometry and distances used for calculating the beam profile and power distribution at the tissue slice. 

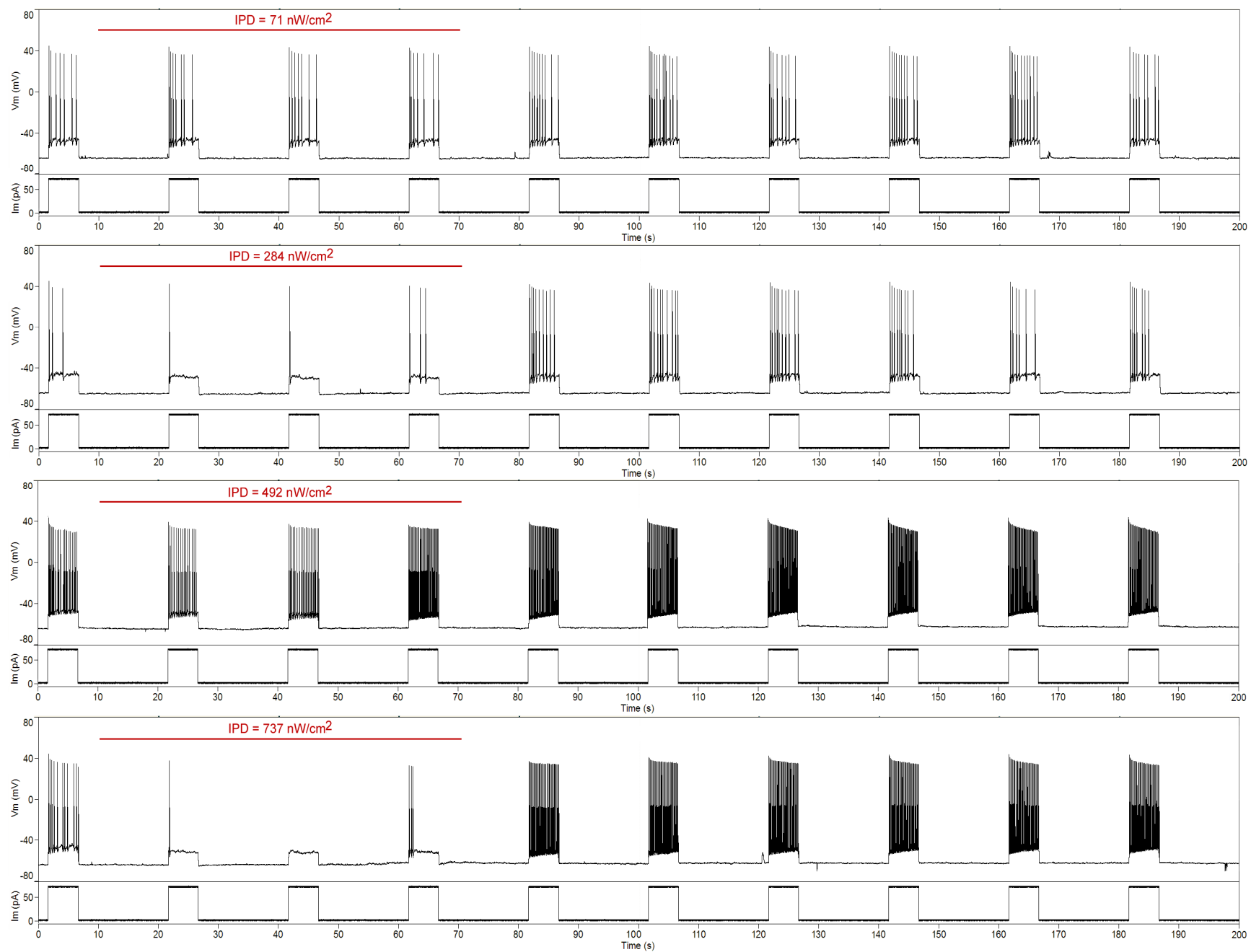

Fig. 14. Sample recording of neuronal activity (membrane voltage, $\mathrm{Vm}$ ) and injected membrane current (Im) at four power levels applied for 1 minute. Incident Power Density (IPD) was calculated according to beam spread and loss in the $2.2 \mathrm{~mm}$ of ACSF fluid above the cortical slice.

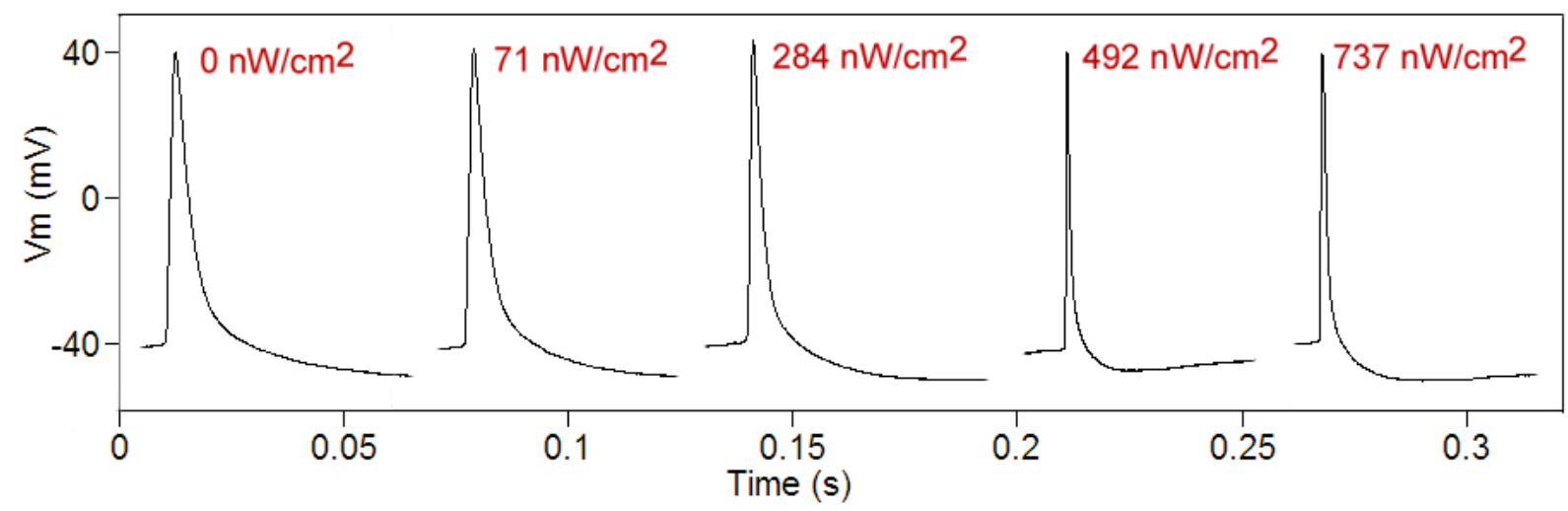

Fig. 15. Typical action potential shapes as a function of RF power density. Exposure was for 1 minute at $60 \mathrm{GHz}$ and corresponds to the four sets of curves in Fig. 14. 


\section{DISCUSSION}

Taken together the described experiments positively show that low levels $\left(<1 \mathrm{~mW} / \mathrm{cm}^{2}\right)$ of millimeter wave power density over short exposure periods (1-2 minutes) can give rise to significant changes in cell activity. It is possible to hypothesize about mechanisms that might produce the observed changes. In all of the experiments, we had to estimate the actual RF power density, which is strongly dependent on the depth of fluid above the cells, all the dielectric interfaces encountered along the RF beam path, and the beam propagation characteristics of the transmitting waveguide. In the patch clamp experiments, RF coupling through the high impedance probe, although unlikely, might be a possible mechanism for concentrating the RF energy. Dielectrophoresis effects in the ACSF media, which contains significant ion content, may also be having an impact. Even modest temperature rise is a strong trigger for membrane depolarization and for changes in action potential firing rates.

Perhaps the strongest indication we have to date of an effect that falls well below that of a rise in the bath temperature due to RF heating is an observed and statistically significant drop in the action potential decay time (from 7 to $1 \mathrm{msec}$ ) and decrease in the membrane input resistance (from 366 to $196 \mathrm{M} \Omega$ ) of the pyramidal neurons at the highest RF power levels (Fig. 15). Even at $185 \mathrm{~mW}$ at the waveguide output port $\left(170 \mathrm{~mW} / \mathrm{cm}^{2}\right.$ at the surface of the ACSF fluid) the temperature rise in the ACSF remained below $3{ }^{\circ} \mathrm{C}$ during the one minute exposure. Comparing the changes in decay time and membrane resistance that we measured over this $3^{\circ} \mathrm{C}$ temperature rise with changes recorded by other researchers on similar cells as a function of broadband bath heating [28], we find that millimeter wave exposure induced $86 \%$ and $46 \%$ decreases in these parameters comparing to changes of only $39 \%$ ( 2.8 to 1.7 msec for decay time) and $35 \%$ (389 to $254 \mathrm{M} \Omega$ for resistance) over a 10 degree rise in bath temperature [28]. This comparison together with the earlier experiments by other groups, suggests a more specific absorption of the millimeter wave RF fields by the exposed cells rather than simple heating of the bath.

\section{SUMMARY}

We have used a number of different experimental techniques to examine the impact of low levels of millimeter wave power density on cell function, most specifically involving changes in the membrane permeability. Taken together the experiments all point to effects that are much greater than those induced by simple thermal heating. Both immortalized epithelial and neuronal cell lines showed similar changes in membrane permeability in vitro. Cortical neurons in brain slices from neonatal rat pups gave the strongest evidence for low level impact of millimeter waves and showed recoverable inhibition (and excitation) in neuronal firing rate as well as correlated changes in rise and decay time and membrane resistance with RF power density. The experiments point to changes in membrane channel opening, which if controllable and proven to have no negative health impact, might be used as a tool for treating peripheral pain, drug delivery, or neuromodulation of central neurological disorders. A lack of high accuracy SAR (specific absorption rate) data for each sample puts large error bars on our experimental values for the tested exposure levels, however even given such uncertainty, the effects we have recorded are clearly observable at levels well below the recommended MPE. We have only begun to evaluate the real-time effects of millimeter waves on cellular functions. We have yet to look at the effects of changing frequency (especially moving to higher frequencies which have higher absorption in tissue), modulation of the incident power at biologically significant rates, pulsed RF fields, sub-second responses of the cells and many other parameters. Further work is certainly needed and the authors hope that the results presented in this paper will catalyze governmental bodies and private foundations overseeing the safety and applications of RF technologies to take a closer look at cellular-level effects of the increasingly wide-spread use of millimeter-wave band devices.

\section{ACKNOWLEDGEMENTS}

The authors are indebted to Caltech Professors Scott E. Fraser of the Biological Imaging Center, and David B. Rutledge of the department of Electrical Engineering for facilities support, programmatic and technical advice and continuous encouragement. Patch clamp measurements would not have been possible without the enthusiastic involvement, perseverance and tremendous skills of Huntington Medical Research Institute's Dr. Michael Harrington and Xianghong Arakaki. This work was supported under limited carryover funds from NIH grant 1 K25 EB00109, two months of discretionary salary support from the NASA Jet Propulsion Laboratory office of the chief scientist, and a significant amount of personal time on the part of both authors. 


\section{REFERENCES}

[1]. G. Lawton, "Wireless HD Video Heats Up," Computer, v. 41, pp.18-20, 2008.

[2]. U.S. Transportation Security Administration, Imaging Technologies, Millimeter Wave Passenger Imaging Tech. Pilot, 2009. http://www.tsa.gov/approach/tech/imaging technology.shtm

[3]. S. Levine "The Active Denial System. A Revolutionary, Non-lethal Weapon for Today's Battlefield," http://handle.dtic.mil/100.2/ADA501865, 1-17, 2009.

[4]. V.V. Vorobyov and R.N. Khramov, "Hypothalamic effects of millimeter wave irradiation depend on location of exposed acupuncture zones in unanesthetized rabbits," Am J Chin Med v. 30, pp. 29-35, 2002.

[5]. S.M. Minasyan, G.Y. Grigoryan, S.G. Saakyan, A.A. Akhumyan, V.P. Kalantaryan, "Effects of the action of microwavefrequency electromagnetic radiation on the spike activity of neurons in the supraoptic nucleus of the hypothalamus in rats," Neurosci Behav Physiol v. 37, pp.175-180, 2007.

[6]. L.I. Kolosova, G.N. Akoev, O.V. Ryabchikova and V.D. Avelev, "Effect of low-intensity millimeter-range electromagnetic irradiation on the recovery of function in lesioned sciatic nerves in rats," Neurosci Behav Physiol, v. 28, pp. 26-30, 1998.

[7]. A.G. Pakhomov, H.K. Prol, S.P. Mathur, Y. Akyel, C.B. Campbell, "Search for frequency-specific effects of millimeter-wave radiation on isolated nerve function," Bioelectromagnetics, v. 18, pp.324-334, 1997.

[8]. A.Y. Sazonov, V.D. Avelev, "Research of biophysical mechanisms of mm-wave effects with use of the bio-technical complex on the basis of skate electroreceptors," Millimeter Waves Med Biol v.28, pp.110-115, 2000.

[9]. S.I. Alekseev, M.C. Ziskin, N.V. Kochetkova and M.A. Bolshakov, "Millimeter waves thermally alter the firing rate of the Lymnaea pacemaker neuron," Bioelectromagnetics v.18, pp. 89-98, 1997.

[10]. S. I. Alekseev and M.C. Ziskin, "Effects of millimeter waves on ionic currents of Lymnaea neurons," Bioelectromagnetics, v. 20, pp.24-33, 1999.

[11]. Alekseev, S. I., Gordiienko, O. V., Radzievsky, A. A. \& Ziskin, M. C., “ Millimeter wave effects on electrical responses of the sural nerve in vivo," Bioelectromagnetics, v.30, 2009.

[12]. S.I. Alekseev and M.C. Ziskin, "Millimeter microwave effect on ion transport across lipid bilayer membranes," Bioelectromagnetics, v.16, pp.124-131, 1995.

[13] A. Ramundo-Orlando, G.P. Gallerano, P. Stano, A. Doria, E. Giovenale, G. Messina, M. Cappelli, M. D'Arienzo and I. Spassovsky, "Permeability changes induced by $130 \mathrm{GHz}$ pulsed radiation on cationic liposomes loaded with carbonic anhydrase," Bioelectromagnetics, v.28, pp.587-598, 2007.

[14] I. Szabo, J. Kappelmayer, S.I. Alekseev and M.C. Ziskin, "Millimeter wave induced reversible externalization of phosphatidylserine molecules in cells exposed in vitro," Bioelectromagnetics, v.27, pp.233-244, 2006.

[15]. A.A. Radzievsky, O.V. Gordiienko, A. Cowan, S.I. Alekseev and M.C Ziskin, "Millimeter-wave-induced hypoalgesia in mice: dependence on type of experimental pain," IEEE Trans Plasma Sci, v. 32, pp.1634-1643, 2004.

[16]. A.A. Radzievsky, O.V. Gordiienko, S.I. Alekseev, I. Szabo, A. Cowan and M.C. Ziskin, "Electromagnetic millimeter wave induced hypoalgesia: frequency dependence and involvement of endogenous opioids," Bioelectromagnetics v.29, pp.284-295, 2008.

[17]. C.K. Chou and J. Andrea (Eds.), "IEEE Standard for Safety Levels With Respect to Human Exposure to Radio Frequency Electromagnetic Fields, 3 kHz to $300 \mathrm{GHz}$," C95.1-2005, 238 pages, 2006.

[18]. E.E. Fesenko, A. Gluvstein, "Changes in the state of water, induced by radiofrequency electromagnetic fields," FEBS Lett, v.367, pp.53-55, 1995.

[19]. O.A. Ponomarev, E.Fesenko, "The properties of liquid water in electric and magnetic fields," Biofizika v.45, pp.389-398, 2000.

[20]. S.J. Kim, B. Born, M. Havenith and M. Gruebele, "Real time detection of protein dynamics upon protein folding by terahertz absorption spectroscopy," Angewandte Chemie International Edition, Wiley Interscience, Engl, v47, pp. 6486-6489, 2008.

[21]. U. Heugen, G.Schwaab, E. Brundermann, M. Heyden, X.Yu, D.M. Leitner and M. Havenith, "Solute-induced retardation of water dynamics probed directly by terahertz spectroscopy," Proc Natl Acad Sci USA, v.103, pp.12301-12306, 2006.

[22]. H. Baranska and A. Labudzinska, "Fermi resonance of $\mathrm{H}_{2} \mathrm{O}$ as a temperature indicator or probe of biological samples in the exciting beam of a laser Raman spectrometer," Applied Spectroscopy, v. 41, no. 6, pp. 1067-1069, 1987.

[23]. N. Kakuta, F. Li, and Y. Yamada, "A method for measurement of water temperature in micro-region using near infrared light," 27th IEEE-EMBS, pp. 3145-3148, 2005.

[24]. J.D. Smith, C. D. Cappa, K. R. Wilson, R. C. Cohen, P. L. Geissler, and R. J. Saykally, "Unified description of temperaturedependent hydrogen-bond rearrangements in liquid water," Proc Natl Acad Sci USA, v.102, pp.14171-14174, 2005.

[25]. V. Pikov and P.H. Siegel, "Remote temperature monitoring of cells exposed to millimeter wave radiation using microscopic Raman spectroscopy," to appear in Engineering in Medicine and Biology Magazine, February 2010.

[26]. M. DiFranco, J. Capote, M. Quinonez, J.L. Vergara, "Voltage-dependent dynamic FRET signals from the transverse tubules in mammalian skeletal muscle fibers," J Gen Physiol v.130, pp.581-600, 2007.

[27]. V. Pikov, X. Arakaki, M. Harrington, S.E. Fraser and P.H. Siegel, "Impacting neuronal activity and plasma membrane properties with low-power millimeter waves, submitted to J. of Neural Engineering, Dec. 2009.

[28]. J.C.F. Lee, J.C. Callaway and R.C. Foehring, "Effects of Temperature on Calcium Transients and Ca2+-Dependent Afterhyperpolarizations in Neocortical Pyramidal Neuron," J Neurophysiol, v.93, pp.2012-2020, 2005. 